\title{
Data repository: Statistical methods
}

The following is written with reference to the Statistical Methods section, and explains more fully the terminology and methods used.

The survivor function, $S(x)$, gives the probability, $P$, of exceedance, such that

$$
S(x)=P(X>x)=1-F(x)
$$

For a sample, $S(x)$ is calculated by rank ordering observations from $X_{i=0}$ to $X_{n}$, where $n$ is the total number of observations, and $S\left(x_{i}\right)=(n-i) / n$. This may then be fitted to a continuous probability distribution survivor function (e.g., Weibull or log-logistic) and be used as a model for forecasting (e.g., Connor et al., 2003).

Probability plots provide a quick assessment of whether data may be described by a particular distribution. Plot axes are constructed by linearizing the cumulative distribution function, $F(x)$. If the data are described by the subject distribution they will plot a straight line, with slope $a$ and intercept $b$. For the Weibull distribution, with repose times $t$, linearization results in the axes:

$$
x=\ln (t) \text { and } y=\ln (\ln (1 /(1-Q(t))))
$$

where $Q(t)(=F(x))$ is the unreliability, and is estimated by Benard's approximation to the median rank: $p=(i-0.3) /(n+0.4)$, where $i$ is the observation rank. By definition $b$ is the $x$-value at an unreliability of 0.632 (for $a=1$ this is equal to the distribution mean).

Linearization of the log-logistic cumulative density function for probability plot construction, with slope $a$ and intercept $b$, gives:

$$
x=\ln (t) \text { and } y=\ln [Q(t) /(1-Q(t))]
$$


Such analysis can be undertaken using any plotting package, such as Excel.

With reference to Kolmogorov-Smirnov $P$-values (Massey, 1951): Since model parameters are estimated from the sample, our $P$-values are conservative; only a high value (perhaps $P>0.9$, for our $n$ ) suggests a model is suitable for describing the sample distribution. The Kolmogorov-Smirnov test does not assume a distribution, and assesses goodness of fit from the maximum deviation between the cumulative distribution function of the data and the model distribution.

\section{Data repository: Figures}

Figure DR1. Survivor functions of best fitting Weibull and log-logistic models against explosion repose interval data from Anak Krakatau. A: 12-13/1/1960; B: 12/1/1960. Insets show histograms of data with the corresponding Weibull and log-logistic probability density functions.

Figure DR2. Survivor functions of best fitting Weibull and log-logistic models against column height data from Anak Krakatau. A: 12-13/1/1960; B: 12/1/1960; C: 13/1/1960. Insets show histograms of data with the corresponding Weibull and log-logistic probability density functions.

Figure DR3. Cumulative distribution functions showing showing explosion data from the Kameni Islands against best-fitting Weibull and log-logistic curves. A: 12/02-25/3/1866, ash explosion frequency; B: 12/02-25/3/1866, steam explosion frequency; C: 12/0225/3/1866, detonation frequency (Schmidt, 1872); D: 14/08-16/09/1925, explosion 
frequency, during the initial paroxysmal stage of the 1925-2928 eruption; E: 23/0914/10/1939, initial vigorous stage, explosion frequency at the Kténas dome; F: 15/1024/11/1939, subsequent quieter stage, explosion frequency at the Kténas dome; G: 22/0223/02/1926, explosion repose intervals during a late eruption period of reduced activity. 
DR2007203 Sebastian Watt, Figure DR1 (figDR1.cdr)
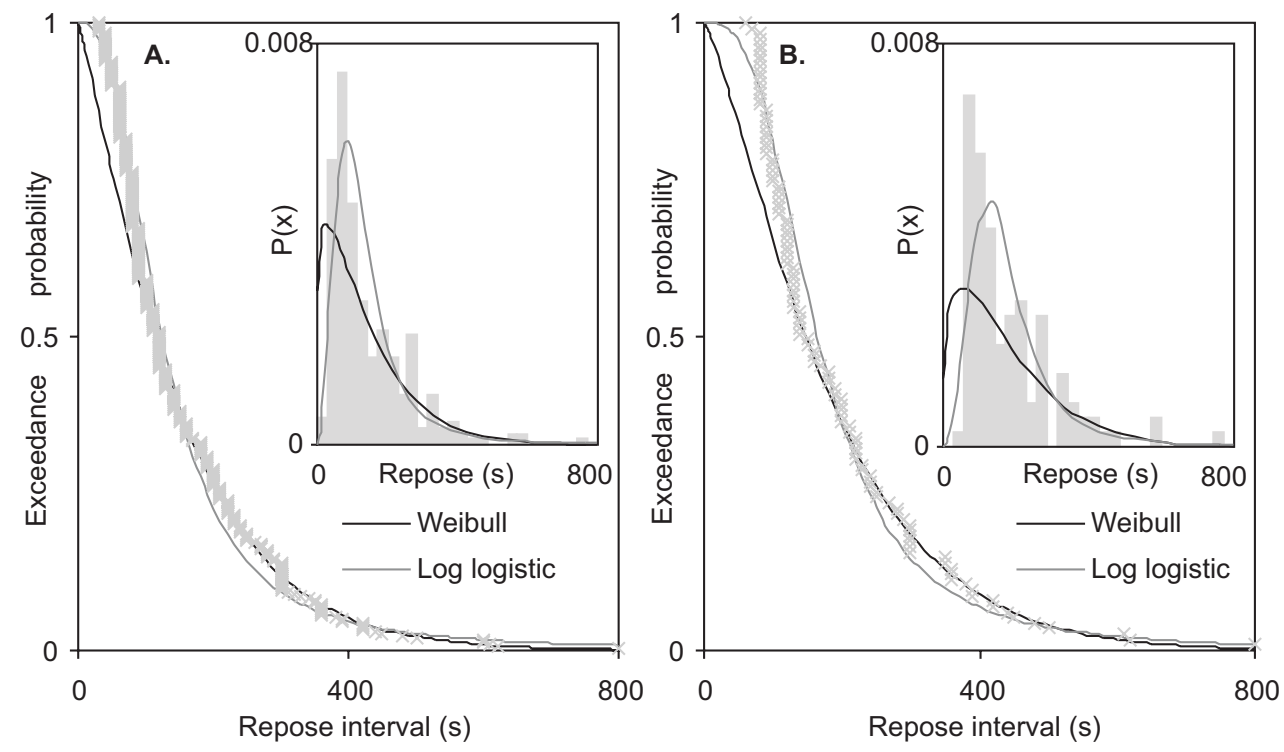
DR2007203 Sebastian Watt, Figure DR2 (figDR2.cdr)
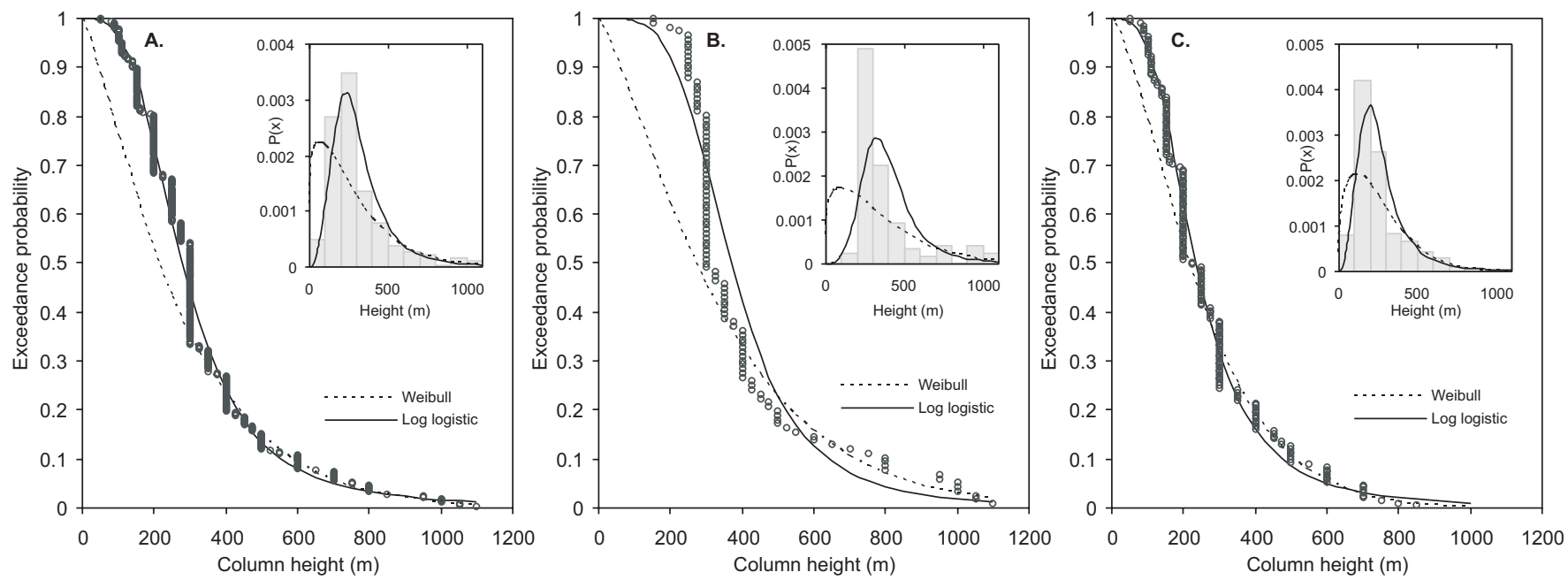

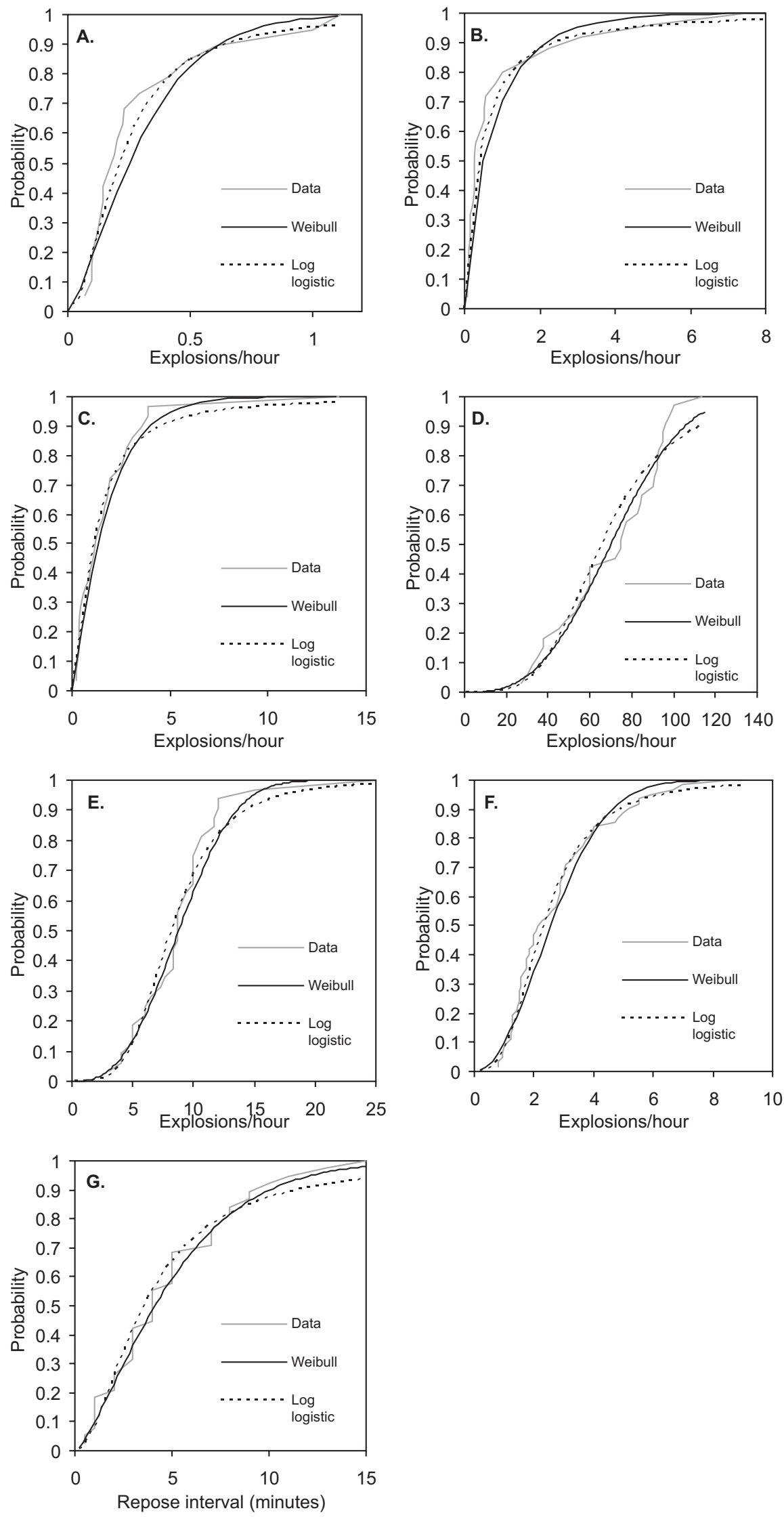